ISSN 2179-345X

Licenciado sob uma Licença Creative Commons

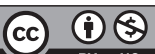

BY NC

\title{
La crisis económica y los mecanismos que se pueden proponer desde la seguridad social ${ }^{1}$
}

\author{
A crise econômica e os mecanismos que se podem \\ propor a partir da segurança social
}

\begin{abstract}
Jordi Garcia Viña
Profesor titular de Derecho del Trabajo y de la Seguridad Social, Universidad de Barcelona - España, e-mail: jordi.garcia@ub.edu
\end{abstract}

\section{Resumen}

Ante la crisis, la gran mayoría de los países industrializados han puesto en práctica amplios conjuntos de políticas activas y de protección, de carácter coyuntural, para contrarrestar los efectos sociales y en el empleo de la recesión económica. La tendencia general es, a diferencia de crisis anteriores, incrementar de manera significativa el esfuerzo en políticas activas de mercado de trabajo a medida que se han ido extendiendo los efectos de la crisis en forma de aumento del desempleo, sobre todo en aquellos países que habían adoptado medidas anticrisis de forma temprana, aunque también se pone de manifiesto que el nivel

1 Este trabajo tiene su origen en la ponencia presentada en el IV Encuentro Internacional de Abogados laboralistas y del movimiento sindical, celebrado en La Habana (Cuba) entre los días 16 a 18 de marzo de 2010. 
global de gasto sigue siendo pequeño en proporción al aumento del paro. A pesar de los enfoques sustancialmente diferentes, los Estados miembros tienen en común problemas cruciales. Como resultado del envejecimiento de la población, el gasto público podría subir vertiginosamente. Para que los trabajadores puedan permanecer más tiempo en activo, es esencial prever los cambios y gestionar con éxito la reestructuración económica. La higiene y seguridad en el trabajo, la organización del trabajo y la formación son dimensiones clave de la calidad del empleo.

Palabras clave: Crisis económica. Mercado laboral. Reestructuración económica.

\section{Resumo}

Diante da crise, a grande maioria dos países industrializados pôs em prática amplos conjuntos de políticas ativas e de proteção, de caráter conjuntural, para neutralizar os efeitos sociais e no emprego da recessão econômica. A tendência geral é, diferente de crises anteriores, aumentar de maneira significativa o esforço em políticas ativas de mercado de trabalho à medida que se vão estendendo os efeitos da crise em forma de aumento do desemprego, sobretudo naqueles países que haviam adotado medidas anticrise de forma adiantada, ainda que também se evidencie que o nível global de gastos continua sendo pequeno em proporção ao aumento do desemprego. Apesar dos enfoques substancialmente diferentes, os Estados-membros têm em comum problemas cruciais. Como resultado do envelhecimento da população, a despesa pública poderia subir vertiginosamente. Para que os trabalhadores possam permanecer mais tempo em atividade, é essencial prever as mudanças e gerenciar com sucesso a reestruturação econômica. A higiene e a segurança no trabalho, a organização do trabalho e a formação são dimensões-chave da qualidade do emprego.

Palavras-chave: Crise econômica. Mercado de trabalho. Reestruturação econômica.

\section{El crecimiento del desempleo y la crisis económica}

La crisis financiera iniciada en el verano de 2007 en Estados Unidos y la posterior recesión económica a escala mundial supusieron el 
inicio de una crisis económica y de empleo de enormes proporciones que difícilmente permite anunciar su salida.

En la Unión Europea el número de personas desempleadas ha aumentado de manera vertiginosa por este motivo. Aunque este incremento afecta a todos los Estados miembros y a todos los colectivos, ya sean hombres o mujeres y/o jóvenes o personas mayores, el impacto ha sido muy diverso y en esta situación pueden influir diversos factores, como las estructuras productivas e institucionales de cada país, así como las medidas económicas y de empleo puestas en práctica.

Conforme a las estadísticas de Eurostat, el número de personas desempleadas se ha incrementado mensualmente desde el mes de marzo de 2008 donde se observan los porcentajes más bajos. España e Italia fueron los primeros países donde el desempleo empezó a incrementarse, aproximadamente a partir de mayo de 2007, seguidos de Irlanda, Luxemburgo, Letonia y Lituania, en la segunda mitad del mismo año y Francia a principios del año siguiente.

Desde el mes de mayo de 2007, el número de personas desempleadas en los países que tienen implantado el Euro se ha incrementado en 3,7 millones hasta alcanzar los 15 millones, mientras que en el mismo período, si se tiene en cuenta todos los países que forman la Unión Europea, el desempleo ha subido en 5,4 millones hasta superar los 21,5 millones $^{2}$ (Gráfico 1).

No sólo hay que tener en cuenta el momento del arranque de la situación de desempleo, sino también la intensidad, que también es muy diferente si se comparan los distintos países. En la mayoría de países, el incremento medio mensual ha sido del 0,1 a 0,2\%; sin embargo, estos incrementos han sido mucho mayores en los países bálticos y en España. Así, por ejemplo, en Estonia, la media ha sido de un punto y Letonia y Lituania han tenido incrementos de 0,6\%. En cuanto a España, ha sido el mercado de trabajo en el que más se ha notado la crisis económica, ya que en un período de 2 años ha habido un incremento de 2,6 millones de

2 Unemployment rate by gender - total. 
desempleados. Estos datos han tenido gran influencia en los porcentajes de desempleo europeos; de hecho, el $20 \%$ de las personas desempleadas en los países de la Unión Europea viven en España (el 29\% si sólo se utiliza los países de la Zona Euro) ${ }^{3}$ (Gráfico 2).

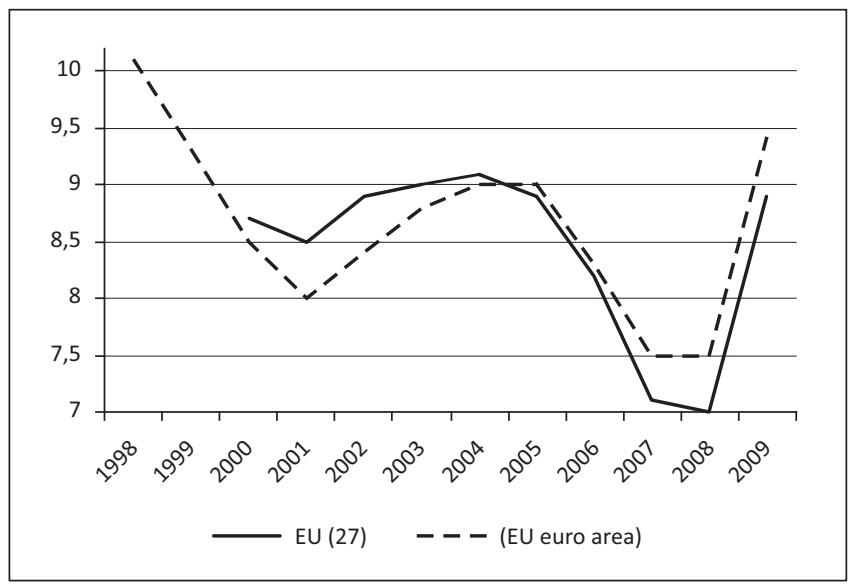

Gráfico 1 - Personas desempleadas

Fuente: EUROSTAT, 2009.

Por otra parte, el desempleo no ha afectado por igual a todos los trabajadores. El peso de la crisis ha recaído principalmente en los trabajadores jóvenes, lo que, unido a que ya constituían el colectivo más afectado por el mismo, ha provocado que sus tasas de desempleo sean especialmente preocupantes, habiendo llegado a alcanzar el 20 por 100 en el tercer trimestre de 2009.

El desempleo masculino, por su parte, ha evolucionado de forma más negativa que el femenino, situándose por encima de éste en el tercer trimestre de 2009, como consecuencia del mayor impacto de la

3 Sharp increase in unemployment in the EU. Statistics in focus, 53/2009. 
crisis en sectores y actividades con mayor concentración de trabajadores varones (Gráfico 3).

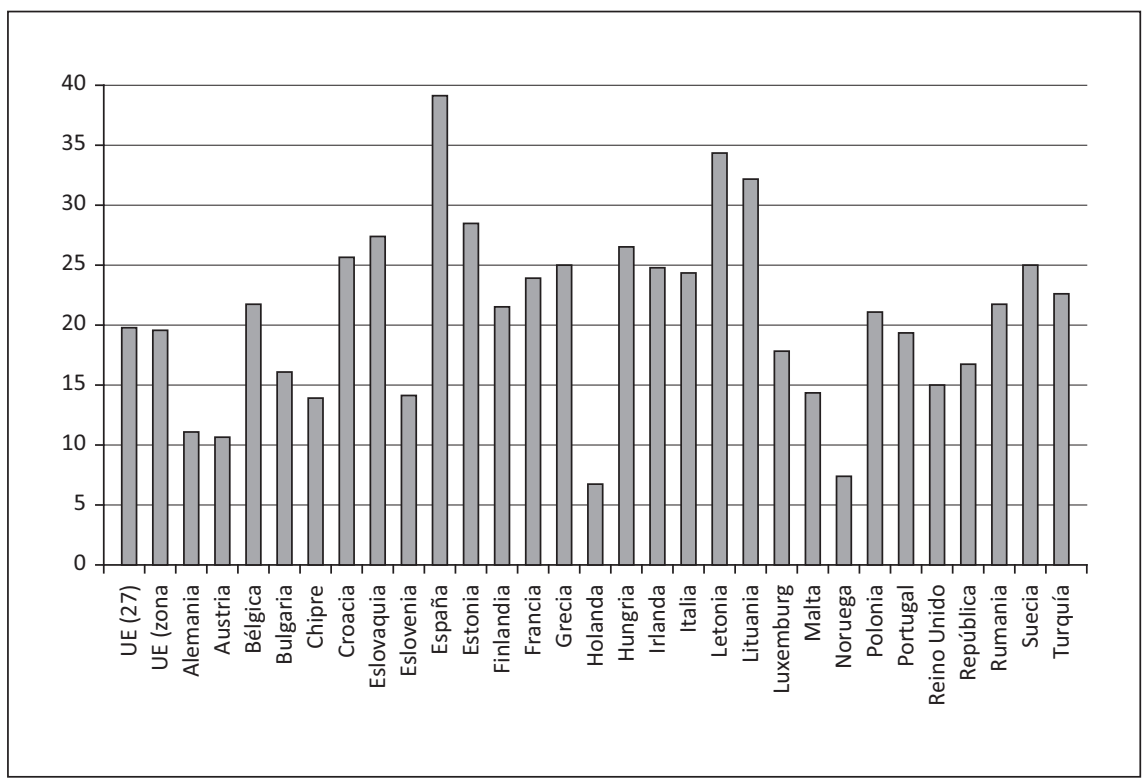

Gráfico 2 - Porcentaje de desempleo de personas jóvenes (menores de 25 años) Fuente: EUROSTAT, 2009.

Por último, los ajustes del empleo ante la crisis han sido mucho mayores entre los trabajadores con contratos temporales y/o niveles formativos más bajos. ${ }^{4}$

En algunas grandes economías que han registrado caídas significativas del PIB, se han dado sin embargo caídas del empleo relativamente reducidas, caso de Alemania, Italia o Reino Unido, a los que se unen otros de menor tamaño como Bélgica, Holanda, Austria, Suecia,

4 The impact of the crisis on employment. Statistics in focus, 79/2009. 
Dinamarca o Finlandia, y, dentro de los países de las últimas ampliaciones, la República Checa, Eslovaquia, Bulgaria, Rumania y Eslovenia. En un país como Francia se ha dado una caída relativamente más moderada del PIB acompañada de una reducción también moderada del empleo. Un caso atípico es el de Luxemburgo, que, pese a haber registrado una caída significativa de su economía, ha experimentado un aumento del empleo. Por el contrario, en otros países con caídas relativamente más bajas del PIB se han producido caídas más drásticas del empleo, como, sobre todo, España, único país comunitario con una caída del empleo muy superior a la del PIB, y, a mucha distancia, Portugal. Por último, se observan países con una caída del PIB pronunciada y una destrucción de empleo también muy importante, como los tres Estados bálticos, Irlanda - con una caída del empleo superior a la del PIB -, o Hungría. ${ }^{5}$

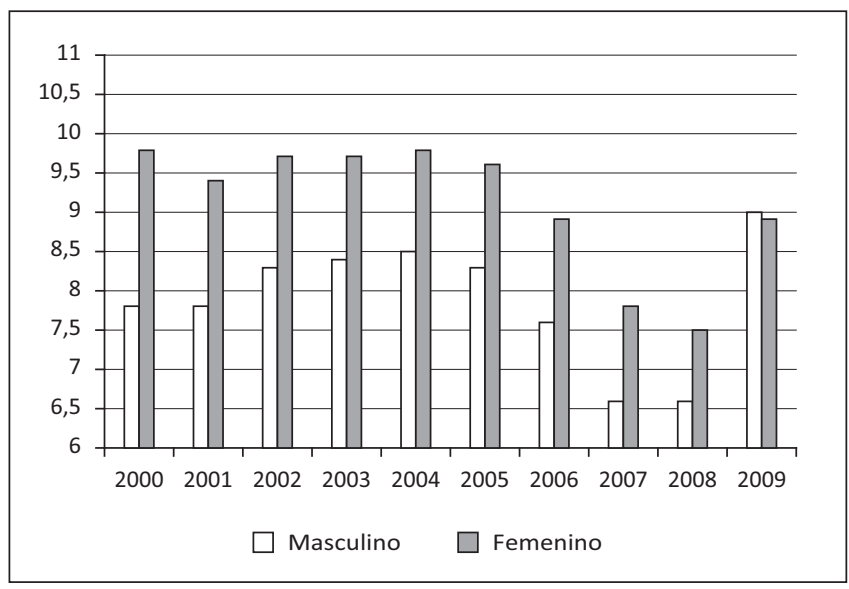

Gráfico 3 - Porcentaje de desempleo por sexo

Fuente: EUROSTAT, 2009.

5 Respuestas a la crisis del empleo en la Unión Europea. Observatorio de relaciones laborales: cauces.

Cuadernos del Consejo Económico y Social, Invierno 2010, p. 21-22. 
En este contexto, aparecen dos incógnitas de difícil resolución: hasta cuándo va a durar la crisis económica y qué incidencia va a tener la recuperación de la crisis con el desempleo.

La Organización para la Cooperación y el Desarrollo Económico estima que el impacto de la crisis en el empleo se va a prolongar más allá del inicio de la recuperación económica. Sus previsiones se cifran en un aumento del paro durante todo el año 2010, a pesar de que desde el primer semestre puede producirse una muy limitada recuperación del PIB. El aumento del paro de larga duración, la pérdida de capital humano a través de la descualificación de los parados de larga duración, hará más difícil la recuperación de los niveles de empleo anteriores a la situación de la crisis, por lo que éstos pueden tardar mucho tiempo en reponerse o pueden incluso no volver a alcanzarse. Puede darse, según sus cálculos, una escalada del paro que puede convertirse en estructural debido al aumento del paro de larga duración o por la salida de la actividad de muchos desempleados. ${ }^{6}$

De la misma manera, la Comisión Europea espera un empeoramiento de las condiciones del mercado de trabajo durante algún tiempo después de que comience a producirse la reactivación del crecimiento económico, por lo que estima que la Unión Europea puede perder todavía 7,5 millones de puestos de trabajo entre 2009 y 2010, alcanzándose en ese año el 10,3 por 100 de tasa de paro. ${ }^{7}$

\section{Mecanismos propuestos para solucionar la crisis}

Ante la crisis, la gran mayoría de los países industrializados han puesto en práctica amplios conjuntos de políticas activas y de protección, de carácter coyuntural, para contrarrestar los efectos sociales y en el empleo de la recesión económica. La tendencia general es, a diferencia de

6 OCDE. Employment Outlook, 2009. Tackling the jobs crisis, p. 12-13.

7 Comisión Europea. La crisis del empleo. Tendencias, respuestas políticas y acciones clave. COM (2009) 649 final, 24 nov. 2009. 
crisis anteriores, incrementar de manera significativa el esfuerzo en políticas activas de mercado de trabajo a medida que se han ido extendiendo los efectos de la crisis en forma de aumento del desempleo, sobre todo en aquellos países que habían adoptado medidas anticrisis de forma temprana, aunque también se pone de manifiesto que el nivel global de gasto sigue siendo pequeño en proporción al aumento del paro. ${ }^{8}$

En un reciente informe, la Organización Internacional del Trabajo ha detectado 32 medidas adoptadas en el ámbito nacional. Las más frecuentemente implementadas han consistido en nueva inversión pública en infraestructuras (el 87 por 100); apoyo a las PYME en forma de ayudas y rebajas fiscales (78 por 100); la mejora del acceso de éstas empresas al crédito (74 por 100); el incremento de las políticas de formación profesional (63 por 100); el aumento de las transferencias en efectivo a las familias (54 por 100); la creación o la ampliación de programas de empleo específicos (52 por 100); el refuerzo de los servicios públicos de empleo (46 por 100); modificaciones en las pensiones de jubilación (44 por 100) o un mayor acceso a las prestaciones de salud (37 por 100).

Concluye el informe que, si bien todos los países han dado mucha prioridad a las nuevas inversiones en infraestructuras como forma de promover la actividad económica y el empleo, los países de nivel económico medio y bajo han realizado un significativo esfuerzo, además, en ampliar la protección social, mientras que los países de ingresos altos han optado más por invertir en las políticas de mercado de trabajo. ${ }^{9}$

En la Unión Europea, la Estrategia de Lisboa para el crecimiento y el empleo se puso en marcha en 2000 como respuesta a los desafíos de la globalización y del envejecimiento. El Consejo Europeo fijó como objetivo de la Estrategia que en 2010 la Unión Europea se convirtiese en la economía basada en el conocimiento más competitiva y dinámica del mundo,

8 OCDE. Employment Outlook, 2009. Tackling the jobs crisis, p. 61.

9 OIT. Proteger a las personas y promover el empleo: un estudio de las respuestas de los países ante la crisis económica mundial en la esfera de las políticas de empleo y de protección social. Septiembre de 2009, p. 46. 
capaz de crecer económicamente de manera sostenible con más y mejores empleos y con mayor cohesión social, pero respetando el medio ambiente. Era evidente que, para mejorar su nivel de vida y mantener su modelo social único, se debía aumentar su productividad y competitividad frente a una competencia global cada vez más fuerte, el cambio tecnológico y el envejecimiento de la población. Además, la agenda de reformas no podía llevarse a cabo solo en el ámbito europeo, porque como muchas áreas implicaban competencias de los Estados miembros, era necesaria una estrecha colaboración entre éstos y la Unión Europea para obtener resultados. También se reconoció por primera vez que las economías de los Estados miembros están intrínsecamente ligadas y que la acción (o falta de acción) de un Estado miembro podría tener consecuencias significativas para la Unión Europea en conjunto.

No obstante, la Estrategia original evolucionó gradualmente hacia una estructura excesivamente compleja con objetivos y acciones múltiples y una división confusa de las responsabilidades y tareas, particularmente entre la Unión Europea y el nivel nacional. Por ello, la Estrategia de Lisboa tuvo que ser relanzada en 2005 tras una revisión intermedia. A fin de tener un mayor sentido de las prioridades, la Estrategia relanzada se centró en el crecimiento y el empleo, estableciéndose una nueva estructura de gobernanza basada en un enfoque de asociación entre los Estados miembros y las instituciones de la Unión Europea.

La propia Comisión Europea constata que la Estrategia de Lisboa ha tenido un impacto positivo en la Unión Europea, aunque no se hayan alcanzado sus objetivos principales (es decir, un nivel de empleo del $70 \%$ y un gasto en I+D del 3\% del PIB). El nivel de empleo de la Unión Europea sólo alcanzó el $66 \%$ en 2008 , antes de volver a caer a consecuencia de la crisis y no se ha logrado superar el desfase de incremento de la productividad con respecto a los principales países industrializados, ya que el gasto total en I+D en la UE, expresado como porcentaje del PIB, apenas mejoró marginalmente (del 1,82\% en 2000 al 1,9\% en 2008). Sin embargo, entiende, es demasiado simplista concluir que la Estrategia fracasó por no haber alcanzado estos objetivos; es más, la Estrategia ha abierto nuevas 
vías al promover acciones comunes para abordar los principales retos a largo plazo de la Unión Europea. ${ }^{10}$

Sin embargo, los vínculos entre la Estrategia de Lisboa y otros instrumentos o estrategias de la Unión Europea, como el Pacto de Estabilidad y Crecimiento, la Estrategia de Desarrollo Sostenible o la Agenda Social, no han sido suficientemente fuertes, de manera que en vez de reforzarse mutuamente, parte de las estrategias han actuado de forma aislada.

Posteriormente, se estableció el programa comunitario para el empleo y la solidaridad social denominado Progress (2007-2013), dividido en cinco secciones que corresponden a cinco ámbitos de acción principales: empleo, protección e integración social, condiciones de trabajo, no discriminación y diversidad e igualdad de género. Este programa tiene por objeto aportar ayuda financiera a la realización de los objetivos de la Unión Europea en el ámbito del empleo y los asuntos sociales. Con un presupuesto de 743 millones de euros, debe financiar actividades de análisis y aprendizaje mutuo, de sensibilización y de difusión, además de ayudas a los principales actores. ${ }^{11}$

De la misma manera, en diciembre de 2008 se aprobó el Plan Europeo de Recuperación Económica (Pere), cuyo objetivo fundamental era dar una respuesta macroeconómica anticíclica a la crisis mediante la acción coordinada de todos los Estados miembros. El Plan constaba de dos pilares:

1) estimular la demanda y generar confianza mediante la inyección masiva de poder adquisitivo en la economía (1,4 por 100 del PIB de la Unión Europea);

2) reforzar la competitividad europea a largo plazo, estableciendo para ello un programa integral para orientar el gasto hacia

${ }^{10}$ Comisión Europea. Documento de evaluación de la Estrategia de Lisboa. 2 de febrero de 2010. SEC (2010) 114 final.

${ }_{11}$ Decisión n. 1672/2006/CE del Parlamento Europeo y del Consejo, de 24 de octubre de 2006, por la que se establece un programa comunitario para el empleo y la solidaridad social - Progress. 
inversiones inteligentes. $Y$ todo ello bajo un principio rector fundamental como es la solidaridad y la justicia social. ${ }^{12}$

De acuerdo con este Plan, la aplicación de políticas activas de inclusión y de flexiguridad integradas, centradas en medidas de activación, de reciclaje profesional y de mejora de cualificaciones, son de vital importancia para fomentar la empleabilidad, lograr la rápida reinserción profesional de los trabajadores que hayan sido despedidos y evitar el desempleo de larga duración. En este contexto, también es importante contar con una protección social adecuada que ofrezca incentivos para trabajar al tiempo que preserva el poder adquisitivo.

Para ello se propone simplificar los criterios para la ayuda procedente del Fondo Social Europeo y adelantar los pagos, de modo que los Estados miembros puedan acceder antes a un importe de hasta 1.800 millones Euros con vistas a reforzar rápidamente, en el contexto de las estrategias de flexiguridad, los programas de activación, especialmente para los trabajadores poco cualificados, que incluyan la asesoría personalizada, el reciclaje profesional intensivo y la mejora de cualificaciones, los periodos de aprendizaje, el empleo subvencionado, y las becas de autoempleo y de creación de nuevas empresas; reorientar sus programas para concentrar su apoyo en los más vulnerables y, en caso necesario, optar por la plena financiación comunitaria de los proyectos durante este periodo; mejorar la supervisión y el desarrollo y mejora de cualificaciones a las vacantes de empleo actuales y futuras; ello se llevará cabo en estrecha cooperación con los agentes sociales, los servicios públicos de empleo y las universidades.

De la misma manera, entiende que los Estados miembros deben plantearse la posibilidad de reducir la fiscalidad sobre el trabajo que se aplica a las rentas más bajas para fomentar la empleabilidad de los trabajadores menos cualificados; así como la introducción de soluciones innovadoras, por ejemplo, cheques de servicios de asistencia a las economías

12 Comisión Europea. Un plan europeo de recuperación económica. COM (2008) 800 final, 26 nov. 2008. 
domésticas y al cuidado de menores, subsidios temporales para la contratación de personas de los grupos vulnerables.

Finalmente, hay que tener en cuenta que la política relativa al empleo en los próximos años en la Unión Europea va a llevarse a cabo por medio de la Estrategia Europea 2020, que sucederá a la actual Estrategia de Lisboa. Este nuevo planteamiento ha de aprovechar los logros obtenidos como asociación para el crecimiento y el empleo y la renovará para hacer frente a nuevos desafíos y beneficiarse de las ventajas derivadas de la respuesta coordinada a la crisis en el Plan Europeo de Recuperación Económica. La Estrategia UE 2020 debe centrarse en aquellos ámbitos políticos clave en los que la colaboración entre la Unión Europea y los Estados miembros puede dar mejores resultados, así como en la mejora de la puesta en práctica, haciendo un mejor uso de los instrumentos disponibles. ${ }^{13}$

La Comisión considera que los factores clave de la estrategia deben ser temáticos y centrarse en las siguientes prioridades:

1) crear valor basando el crecimiento en el conocimiento. En un mundo en el que la innovación, tanto en los productos como en los procesos, marca la diferencia, se reforzarán las oportunidades y la cohesión social, aprovechando el potencial que encierran la educación, la investigación y la economía digital;

2) potenciar el papel de los ciudadanos en sociedades inclusivas. La adquisición de nuevas capacidades que estimulen la creatividad y la innovación, el desarrollo del espíritu empresarial y una transición fluida entre diferentes empleos serán elementos cruciales en un mundo que ofrecerá más empleos a cambio de una mayor capacidad de adaptación;

3) crear una economía competitiva, conectada y más respetuosa del medio ambiente. La Unión Europea ha de ser capaz de competir

${ }^{13}$ Comisión de las Comunidades Europeas - Documento de trabajo de la Comisión. Consulta sobre la futura estrategia UE 2020. Bruselas, 24 de noviembre de 2009. COM (2009) 647 final. 
de manera más efectiva y de mejorar su productividad consumiendo menos recursos y energías no renovables, y haciéndolo de manera más eficiente, en un mundo en el que los precios serán altos y cada vez será más reñida la competencia para obtenerlos. De esta forma se estimulará el crecimiento y se contribuirá a la consecución de nuestros objetivos medioambientales. Ello redundará en beneficio de todos los sectores de la economía, desde las industrias manufactureras tradicionales hasta las empresas emergentes de alta tecnología. La modernización y la interconexión de las infraestructuras, la reducción de la carga administrativa y la aceleración de la penetración de las innovaciones en el mercado contribuirán igualmente a la consecución de este objetivo.

Si se analizan las principales medidas de respuesta a la crisis, en términos de esfuerzo de inversión realizado en los países miembros de la Unión, se observan cuatro grandes bloques de mecanismos. En primer lugar, la prioridad otorgada por los gobiernos a los estímulos para la reactivación económica y para la demanda de trabajo en forma de medidas económicas generales de ayuda, garantías públicas o reducciones fiscales, a las que se pueden sumar los programas de apoyo específicos para sectores económicos en dificultades. En segundo lugar, un bloque de medidas de estímulo corresponde a las nuevas inversiones en infraestructuras con los mismos objetivos de sostenimiento de la demanda y de la actividad económica general. En tercer lugar, la mayoría de los Estados han adoptado algún tipo de medidas de mejora de la protección de los parados, refuerzo de los servicios públicos de empleo, programas de trabajos públicos o apoyo a medidas de flexibilidad como, sobre todo, programas de reducción del tiempo de trabajo. Por último, muchos países han introducido medidas de formación y de cualificación de los trabajadores y de refuerzo de la búsqueda de empleo y la colocación de los desempleados, aunque ningún país ha concentrado el esfuerzo en esas políticas en términos de recursos (VOGLER-LUDWIG, 2009). 


\section{La Seguridad Social como medida de resolución de la crisis}

La Seguridad Social puede ser un instrumento más en manos de los gobiernos europeos a la hora de resolver la crisis. Las posibilidades son varias y complejas y hay que tener cuidado porque, en caso de equivocación, los efectos pueden ser perversos y ocasionar importantes perjuicios para amplias capas de la población.

Es evidente que uno de los mecanismos para resolver esta situación puede ser controlar la financiación del gasto público derivado de la Seguridad Social.

Según un estudio realizado por la Asociación Internacional de Seguridad Social, los ingresos de la seguridad social han disminuido significativamente a causa de la crisis: se ha producido una pérdida de ingresos, debida, sobre todo, a la disminución de las contribuciones y de los ingresos derivados de las inversiones, a la caída de las subvenciones de los gobiernos y de las subvenciones cruzadas entre regímenes, y al incremento de los impagos y las reservas de la seguridad social también han disminuido.

Los principales fondos de la seguridad social asociados a programas públicos de seguridad social han obtenido un rendimiento negativo de las inversiones y una pérdida total estimada en 225.000 millones de dólares estadounidenses para 2008. Para algunos fondos de la seguridad social, la pérdida representa el equivalente a cinco años de ingresos derivados de las inversiones y a cerca del $25 \%$ del valor neto de los activos del fondo. Según un informe de la OCDE, ${ }^{14}$ la preocupación de los trabajadores en cuanto a la disminución del valor de los ahorros de las pensiones privadas es justificada. De acuerdo con las estimaciones de esta organización, la pérdida de activos de pensiones privadas registrada durante el año 2008, que fue de 5 trillones de dólares hasta octubre de 2008, ha aumentado hasta 5,4 trillones de dólares estadounidenses. La tasa de retorno media de los fondos de pensiones ha registrado un valor medio negativo del $23 \%$ anual. ${ }^{15}$

${ }^{14}$ OCDE. Private Pensions Outlook, 2008.

15 OIT. Global Employment Trends, mayo de 2009. 
Además de la caída de ingresos y reservas, el gasto de la seguridad social ha crecido considerablemente debido al incremento de la demanda de prestaciones por desempleo, vivienda y asistencia social. Hay indicios de que los cuidados de salud están aumentando como efecto derivado de la crisis.

En consecuencia, muchos programas de seguridad social se están viendo afectados por problemas financieros a corto y medio plazo. Por ejemplo, la mejora de las prestaciones o la disminución de la carga que representan las contribuciones sociales para las empresas podrían provocar serios desequilibrios económicos entre los programas y las organizaciones de seguridad social. Medidas tales como las encaminadas a aumentar las prestaciones o a congelar los incrementos programados de las tasas de contribución, o incluso a reducir las tasas actuales, pueden llevar a un aumento de los ingresos disponibles de las personas o a una mejora del flujo de caja de las empresas a corto plazo, pero también pueden generar una disminución de los ingresos de la seguridad social. ${ }^{16}$

La viabilidad de las pensiones depende de su capacidad para cubrir los objetivos sociales de forma continua manteniendo al mismo tiempo otros importantes objetivos políticos, como son unas finanzas públicas saneadas y la justicia entre las generaciones. La viabilidad no puede venir de uno o varios elementos aislados. Unos altos índices de crecimiento económico y de aumento del empleo son esenciales y contribuirán mucho a la viabilidad. Simultáneamente, las reformas de los propios regímenes de pensiones ayudarán a hacerlos viables, sobre todo de cara a las incertidumbres que plantea la cuestión a largo plazo. Esas reformas pueden abarcar tanto los parámetros de los regímenes de pensiones públicos como la propia estructura del sistema de pensiones, es decir, las cotizaciones de los tres niveles.

Los temas relacionados con la reforma de las pensiones han tenido desde hace tiempo un lugar preferente en las agendas de muchos Estados miembros. Varios Estados miembros han emprendido ya reformas que

${ }^{16}$ AISS. Respuestas de la seguridad social a la crisis financiera, 23 jun. 2009. 
pueden incluir los tres niveles del sistema. Las reformas de los regímenes públicos de pensiones se centran principalmente en controlar el crecimiento del gasto, mientras que en muchos casos es preciso mejorar los regímenes de pensiones profesionales e individuales para que puedan tener un papel más importante en los ingresos de las personas de edad avanzada. Dado que los sistemas son diferentes en cada Estado miembro, no es aconsejable ni adecuado dar una respuesta para toda la Unión Europea. Sólo será posible garantizar unos regímenes viables y unas pensiones seguras cuando cada uno de los Estados miembros haya definido los cambios que requiere su política actual y establecido el consenso necesario para su aplicación.

A pesar de los enfoques sustancialmente diferentes, los Estados miembros tienen en común problemas cruciales. Como resultado del envejecimiento de la población, el gasto público podría subir vertiginosamente. Si no se efectúan reformas, en 2030 el gasto de los sistemas públicos de pensiones alcanzará en algunos Estados miembros entre el 15\% y el $20 \%$ del PIB. ${ }^{17}$

Los compromisos en concepto de pensiones sin un capital de respaldo pueden equivaler en algunos casos al 200\% del PIB. ${ }^{18} \mathrm{Un}$ importante crecimiento en el número de pensionistas requiere forzosamente que se incrementen los recursos aportados por la población activa. Las tasas de empleo y los niveles de productividad de la población activa serán, por tanto, importantes factores determinantes de las condiciones de vida de los pensionistas. Todos los Estados miembros tienen la necesidad de modernizar o ajustar los regímenes de pensiones y las prácticas del mercado de trabajo para que se apoyen mutuamente y contribuyan mejor a fomentar el crecimiento económico y la cohesión social.

17 Comunicación de la Comisión, de 12 de octubre de 2006, «El futuro demográfico de Europa: transformar un reto en una oportunidad» [COM (2006) 571 final - no publicada en el Diario Oficial].

${ }^{18}$ Comunicación de la Comisión, «Hacia un mercado único de sistemas complementarios de pensiones», COM (1999) 134 final. 
La modernización y reforma de los regímenes de pensiones es un proceso complejo que integra una amplia gama de intereses y protagonistas. De él dependen las condiciones de vida de un sector de la población numeroso y potencialmente vulnerable. Es preciso respetar expectativas legítimas y mantener un equilibrio justo y viable entre derechos y obligaciones. Las reformas deben poder afrontar los retos que se avecinan, ser creíbles, transparentes y claras en su impacto y conceder suficiente tiempo a la población para adaptarse. El proceso de reforma exige que todos los protagonistas participen y estén dispuestos a cambiar.

Por lo tanto, es necesario estimular un esfuerzo conjunto para hallar modos prácticos de hacer viable la prestación de pensiones adecuadas, manteniendo al mismo tiempo unas economías públicas saneadas y una justicia entre las generaciones.

Hay que tener en cuenta que los regímenes de pensiones aportan la mayoría de los recursos de que disponen las personas de edad avanzada. Entre todas las ayudas que se ofrecen a los jubilados, las pensiones ocupan el mayor segmento, y la principal fuente de ingresos en concepto de pensiones son los regímenes públicos. Las prestaciones de vejez y de supervivencia representan, en conjunto, casi el $45 \%$ del gasto total de protección social en la UE y alrededor del $12 \%$ del PIB. La mayor parte del gasto en concepto de vejez y de supervivencia procede de regímenes de pensiones obligatorios, y se financia con las cotizaciones a la seguridad social y los impuestos. Las personas de edad avanzada, al ser también los principales beneficiarios de la asistencia sanitaria y de larga duración, constituyen el segmento de la sociedad más dependiente de los regímenes de protección social. El segundo gasto más importante en protección social es la asistencia sanitaria, que representa más del 7\% del PIB ${ }^{19}$ (Gráfico 4).

${ }^{19}$ Comunicación de la Comisión al Parlamento Europeo, al Consejo, al Comité Económico y Social Europeo y al Comité de las Regiones, de 29 de abril de 2009-Abordar los efectos del envejecimiento de la población de la UE (Informe de 2009 sobre el envejecimiento demográfico) [COM (2009) 180 final - no publicada en el Diario Oficial]. 


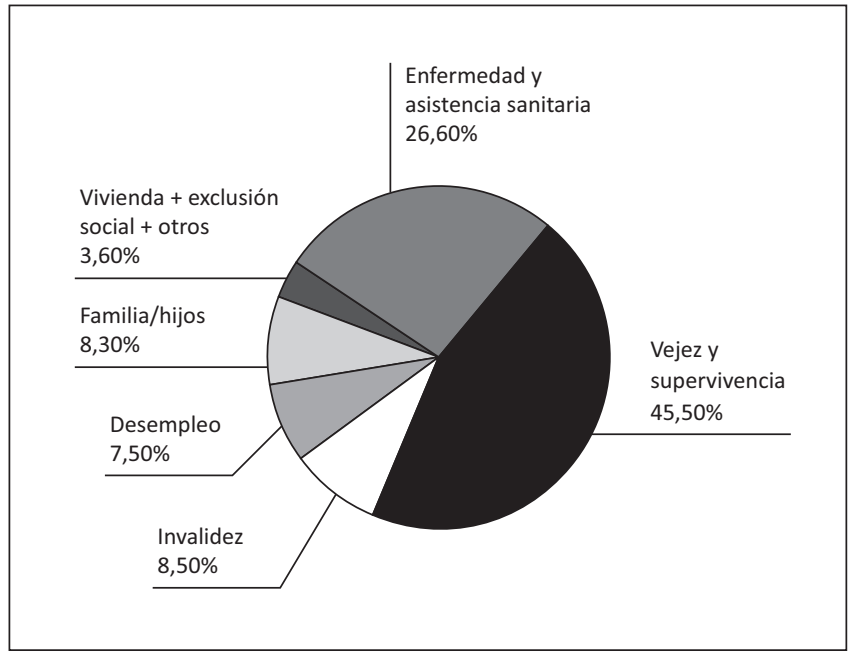

Gráfico 4 - Prestaciones sociales por funciones

Fuente: Dados de investigación.

Como se puede comprobar son las pensiones de vejez y supervivencia las que suponen una mayor carga a los presupuestos de la Seguridad Social y, según la evolución observada en los últimos datos estadísticos, este proceso es continuado. De esta manera, se puede afirmar que la financiación de las pensiones es una de las áreas problemáticas más importante de la sociedad actual (BAREA; GONZALEZ-PARAMO, 1996, p. 14).

Durante las últimas décadas, los regímenes europeos de pensiones han logrado con notable éxito sus objetivos sociales. Las pensiones públicas han supuesto un cambio para la seguridad de los ingresos y la situación económica de los pensionistas de edad avanzada y les ha permitido ser independientes y disfrutar de un largo período de ocio después de sus carreras laborales.

El riesgo de pobreza en la vejez, que antiguamente estaba muy extendido, se ha reducido enormemente, al igual que el número de personas que tienen que depender de sus descendientes o de subsidios sociales 
sujetos a la prueba de recursos. Esto se ha logrado gracias a los regímenes de pensiones, a pesar de un notable crecimiento de la esperanza de vida y del número de pensionistas en las últimas décadas.

El mayor y más claro de los retos a largo plazo a que se enfrentan los actuales regímenes de pensiones es el envejecimiento demográfico. No obstante, la necesidad de reformar los regímenes de pensiones no sólo viene del cambio en el equilibrio demográfico. Hay una delicada interacción, que no se debería olvidar, entre la viabilidad financiera de las pensiones, el desarrollo económico y el empleo. Por un lado, el crecimiento económico y del empleo puede abrir nuevas oportunidades; es fundamental aumentar el número de personas que trabajan para estabilizar o incluso reducir los coeficientes de dependencia económica. Por otra parte, los regímenes de pensiones tienen que ser más eficaces, tanto en el logro de sus objetivos sociales como en el apoyo a los objetivos europeos de empleo y crecimiento.

En las próximas décadas, el número de personas de la tercera edad va a aumentar mucho en relación con la población en edad laboral. No hay políticas razonables capaces de contrarrestar este cambio en la estructura demográfica de la Unión Europea. Sin embargo, lo que afecta a la viabilidad de las pensiones no es el coeficiente de dependencia de la tercera edad, sino el coeficiente de dependencia económica.

El aumento de la esperanza de vida gracias a la mejora del bienestar, los avances de la medicina y el descenso de las tasas de fertilidad son los vectores de una tendencia secular al envejecimiento de la población. Esta tendencia no es nueva y va a continuar. Los avances de la medicina no dan señales de hacerse más lentos, y las previsiones pasadas han tendido a subestimar el incremento de la esperanza de vida. Las tasas de fertilidad actuales están bastante por debajo del nivel que se precisa para la renovación completa de toda la población. Podrían subir si se eliminaran algunos de los obstáculos (principalmente en lo que respecta al mercado de trabajo, la vivienda y el cuidado de los niños) que disuaden de formar una familia y tener hijos. Las tasas de fertilidad tienden a ser más altas en los Estados miembros que aplican una política decidida para 
conciliar la familia y la vida laboral. Sin embargo, siguen siendo demasiado bajas como para evitar el envejecimiento de la población.

El principal cambio de las próximas décadas será la llegada a la edad de jubilación de la numerosa generación del boom, nacida después de la Segunda Guerra Mundial. Este aumento de la población se verá reflejado inicialmente en una población activa de más edad, posteriormente, a partir de 2010, en un fuerte incremento del número de beneficiarios de pensiones y más tarde en un aumento de las necesidades de asistencia sanitaria y cuidados de larga duración.

El coeficiente de dependencia de la tercera edad aumentará considerablemente en los próximos 30 a 40 años. Según las proyecciones demográficas a largo plazo de Eurostat, el número de personas en edad laboral por pensionista se reducirá a la mitad de aquí a 2050, pasando de 3,5 a 1,8 en la Unión Europea. El efecto demográfico del boom de la natalidad de la postguerra empezará a decrecer hacia 2030 y no se espera que desaparezca hasta mediado el siglo.

El número de personas de edad crecerá de tal modo que ni un rápido aumento de la fertilidad ni ningún nivel realista de inmigración pueden detener el incremento del coeficiente de dependencia de la tercera edad. Los cambios en la tasa de fertilidad sólo empiezan a influir en el mercado laboral veinte años después de producirse. No obstante, la inmigración puede ayudar a aumentar el nivel de empleo, pero su impacto positivo depende de la medida en que los inmigrantes puedan estar suficientemente integrados en el mercado de trabajo (Gráfico 5).

Sin embargo, la viabilidad de las pensiones no depende del coeficiente demográfico de dependencia, sino de sus efectos para el coeficiente de dependencia económica (que es significativamente mayor) y las transferencias. Por ello, es mucho más procedente para evaluar la viabilidad futura de los regímenes de pensiones tomar en consideración el número real de personas empleadas en relación con las que no trabajan. Actualmente, el coeficiente de dependencia económica (el número de personas de 20 años o más que no trabajan en relación con el total de la población empleada) es de 0,86 en la Unión Europea, lo que significa que hay 
casi tantas personas en edad activa desempleadas como empleadas. Cerca de un $60 \%$ de los adultos que no trabajan están por debajo de los 65 años, y de éstos son muchos los que se benefician de prestaciones de diversos tipos. Es de temer que el coeficiente de dependencia económica se vea negativamente afectado por el aumento del coeficiente de dependencia de la tercera edad pero, si se reduce el número de las personas inactivas entre la población en edad laboral, será posible aliviar la carga financiera que para los activos supondrá el envejecimiento. Por lo tanto, es necesario saber en qué medida será posible contrarrestar el impacto del envejecimiento en los coeficientes de dependencia económica subiendo los índices generales de empleo ${ }^{20}$ (Gráfico 6).

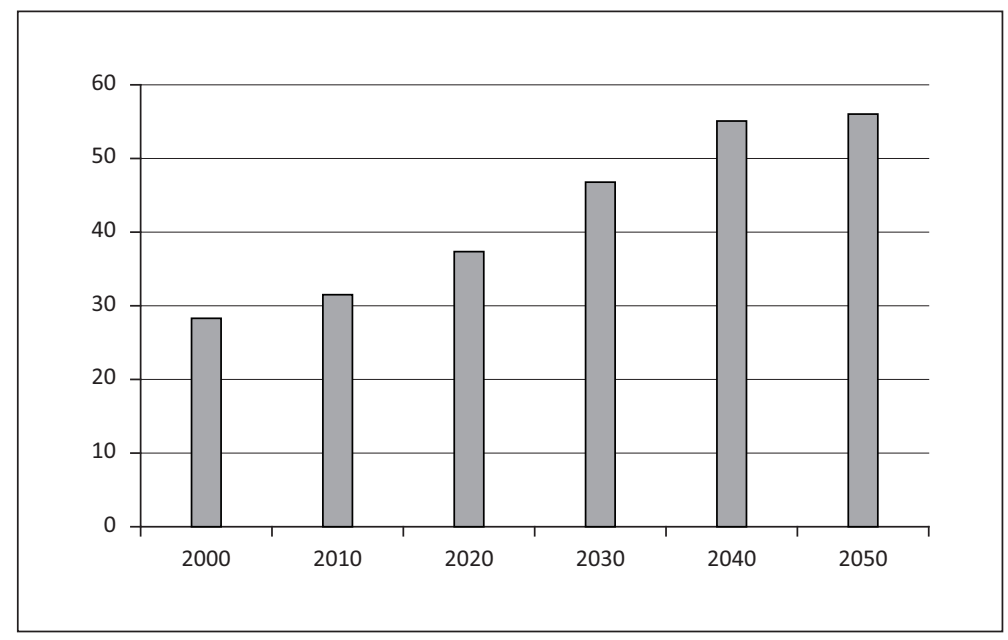

Gráfico 5 - Proyecciones básicas de los coeficientes de dependencia medios de la tercera edad en Unión Europea

Fuente: Dados de investigación.

${ }^{20}$ Comunicación de la Comisión al Consejo, al Parlamento Europeo y al comité económico y social. Evolución futura de la protección social desde una perspectiva a largo plazo: pensiones seguras y viables. Bruselas 11 de octubre de 2000. COM (2000) 622 final. 


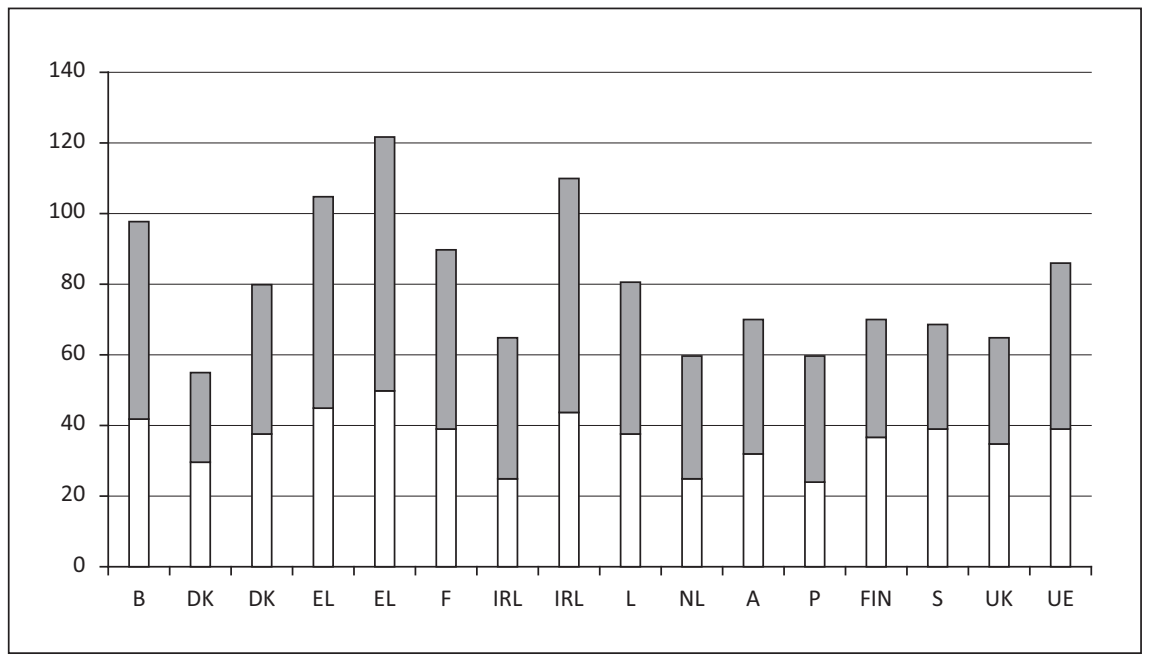

Gráfico 6 - Coeficientes de dependencia económica Fuente: Dados de investigación.

\section{¿Es una solución retrasar la edad de jubilación?}

El bajo nivel de empleo de los trabajadores de más edad en Europa es un despilfarro de oportunidades en la vida de las personas y de potencial social. A medida que crece la esperanza de vida y que la generación del boom demográfico provoca una enorme expansión de la franja de edad entre 55 y 64 años, esta pérdida se hace cada vez más insostenible. Debido al continuo aumento de la longevidad, las personas tienen más oportunidades de desarrollar su potencial durante una vida más larga. Sin embargo, si no tienen suficientes posibilidades de participar en el empleo, sus condiciones de vida no alcanzarán plenamente su nivel potencial.

Para la economía en su conjunto, el aumento de la participación y de las tasas de empleo de las personas de más edad son cruciales para aprovechar plenamente la oferta de mano de obra a fin de apoyar el crecimiento económico, los regímenes fiscales y de prestaciones y los sistemas 
de protección social, que deben incluir unas pensiones correctas, ante la previsible reducción de la población en edad laboral. En 2030, más de 110 millones de personas superarán los 65 años en EU-25, cuando eran 71 millones en 2000, mientras que la población en edad laboral se quedará en 280 millones, de los 303 millones actuales. Esto tiene una repercusión directa en la capacidad para apoyar el crecimiento económico a largo plazo. A menos que puedan lograrse tasas de empleo superiores al objetivo general del $70 \%$ y mejore mucho la productividad, el crecimiento del PIB per cápita en la Unión Europea se ralentizará significativamente entre 2010 y 2030.

Las prácticas en el lugar de trabajo y el mercado deben reflejar la realidad de que las personas de más de 50 años constituirán una proporción significativamente mayor de la población activa potencial, al tiempo que irá disminuyendo el número de jóvenes que se incorporan a ella. Es una prioridad urgente cambiar las actitudes y las expectativas de los trabajadores y empresarios. A menos que se adopten medidas drásticas en consonancia con la estrategia europea de empleo y con las orientaciones y recomendaciones generales de política económica, no podremos alcanzar los objetivos de la Unión Europea en materia de empleo.

Además, hay que tener en cuenta que la edad media de salida del mercado de trabajo varía considerablemente según los países, oscilando entre los 56,9 años de Polonia y los 63,2 de Suecia. Los Estados miembros más rezagados son los mismos que presentan las tasas de empleo más bajas. La diferencia en la edad media de salida de hombres y mujeres es pequeña, a pesar de la considerable divergencia de las tasas de empleo. Esto se debe al hecho de que muchas más mujeres que hombres no han formado parte nunca de la población activa, aunque el reducido número de mujeres que están en el mercado de trabajo tiende a abandonarlo a edades similares a las de los hombres.

Sin embargo, las cifras sobre edad de salida también deben analizarse teniendo en cuenta el tiempo permanecido en el mercado de trabajo. Mientras a escala de la Unión Europea la edad media de salida de los trabajadores poco cualificados está tres años por debajo de la de los 
altamente cualificados, esto se compensa al haber comenzado su vida laboral tres años antes.

A pesar de que los progresos en toda la Europa de los Quince han sido lentos en la década de los noventa, más recientemente se ha acelerado el aumento de la tasa de empleo de los trabajadores de más edad, hasta superar el de la tasa de empleo de todos los trabajadores en edad laboral. En los nuevos Estados miembros, las tasas de empleo de la población en edad de trabajar y de los trabajadores de más edad han empeorado significativamente a finales de los noventa. La posterior mejora de la tasa de empleo de los trabajadores de más edad sólo ha compensado este deterioro parcialmente, mientras que la tasa de empleo global siguió empeorando.

Está probado que es posible incrementar la participación en el mercado de trabajo y las tasas de empleo de los trabajadores de más edad cuando existen las condiciones y políticas del mercado de trabajo correctas: el aumento del empleo de estos trabajadores y el retraso de su edad media de salida del mercado de trabajo no son sólo debidos al crecimiento general del empleo en los Estados miembros. Se ha logrado un crecimiento importante de las tasas de empleo de los trabajadores de más edad incluso en Estados miembros con un lento aumento general del empleo. Se requieren unas buenas condiciones macroeconómicas para mantener niveles elevados de empleo, pero para que las carreras profesionales puedan ser largas deben darse determinadas condiciones en el mercado de trabajo. ${ }^{21}$

\section{Incentivos financieros adecuados}

Mientras alrededor de un tercio de las personas inactivas de entre 55 y 64 años mencionan la jubilación normal como principal motivo para

${ }^{21}$ Comunicación de la Comisión al Consejo, al Parlamento Europeo, al comité económico y social y al comité de las regiones. Aumentar el empleo de los trabajadores de más edad y retrasar su salida del mercado de trabajo. Bruselas 3 de marzo de 2004. COM (2004) 146 final. 
abandonar su último empleo o negocio, más del 20\%, lo que representa una media de unos 3 millones al año, señala que el principal motivo fue la jubilación anticipada. Estos resultados ponen de manifiesto la importancia de reformar las disposiciones sobre jubilación anticipada y, más en general, de revisar los incentivos financieros y de aplicar debidamente otros regímenes de prestaciones (como las de desempleo de larga duración, enfermedad o discapacidad prolongada, que pueden ofrecer vías alternativas al abandono del mercado de trabajo) a fin de lograr que resulte rentable permanecer en el mercado de trabajo.

\section{Buenas condiciones de higiene y seguridad en el trabajo}

El segundo motivo en importancia es la enfermedad o discapacidad prolongada. Por otra parte, la tasa de prevalencia normalizada de los problemas de salud relacionados con el trabajo aumenta con la edad. Esto refuerza el papel crucial de las condiciones de higiene y seguridad para el bienestar de los trabajadores de más edad y sus posibilidades de seguir en el mercado. Estos aspectos también son importantes a la hora de evitar pérdidas involuntarias de productividad laboral como resultado de un aumento de los días de baja.

\section{Formas flexibles de organización del trabajo}

Las formas flexibles de organización del trabajo son otro componente de las condiciones de trabajo que puede favorecer el mantenimiento en activo de los trabajadores de más edad. Las encuestas nacionales demuestran que un porcentaje significativo de los trabajadores de más edad preferirían retirarse gradualmente, principalmente por motivos de salud vinculados al envejecimiento. Entre 2001 y 2002, los fuertes aumentos de las tasas de empleo de los trabajadores de más edad estuvieron asociados, en la mayoría de los Estados miembros, a incrementos del porcentaje de 
trabajadores a tiempo parcial. El 36\% de la creación de empleo para trabajadores de más edad entre 1997 y 2002 corresponde a puestos a tiempo parcial, lo que hizo aumentar la proporción de estos trabajadores a tiempo parcial. Si los trabajadores deciden reducir progresivamente sus horas de trabajo a lo largo de un período, la jubilación se convierte en un proceso, en lugar de un suceso. La jubilación a tiempo parcial es una opción que merece más atención de la que actualmente se le presta.

Los distintos empleos y actividades presentan diferentes niveles de carga física y mental. A medida que los trabajadores tienen más edad, deben tener la oportunidad de ocupar los puestos más acordes con sus aptitudes intelectuales y físicas, y debe dárseles opción a elegir un trabajo a tiempo parcial o, al menos, una reducción de su horario de trabajo.

Los trabajadores también deben considerar el empleo por cuenta propia como una alternativa al trabajo a tiempo parcial. Esta opción resulta especialmente idónea para los trabajadores a partir de 50 años: en 2002, el empleo por cuenta propia en este grupo de edad aumentó alrededor de un $3 \%$, frente al aumento de menos del 0,3\% que registró el de los trabajadores en edad de máximo rendimiento.

\section{Acceso continúo a la formación}

La permanencia en el empleo y los niveles de empleo de los trabajadores de más edad están estrechamente relacionados con el nivel de formación que reciben y con su nivel educativo inicial. También hay un marcado paralelismo entre el nivel educativo alcanzado y la calidad del empleo, atendiendo a las condiciones salariales y laborales de que disfruta el trabajador. No hay pruebas de que los trabajadores de más edad sean más o menos productivos que los de otras categorías. El potencial de productividad de estos trabajadores no se ve afectado por su edad, sino porque sus aptitudes quedan anticuadas, y esto se puede corregir mediante la formación [9]. La experiencia de los trabajadores de más edad hace que sean un valioso recurso para las empresas. La formación también 
representa una oportunidad para que los trabajadores actualicen sus aptitudes y refuercen su situación en el mercado laboral, particularmente desarrollando las capacidades necesarias para aprovechar plenamente las tecnologías de la información y la comunicación.

En este contexto, como ocurre también para otras categorías de edad, es importante reconocer que el reto no es sólo aumentar la participación en la formación profesional, sino también mejorar los niveles generales de conocimientos y competencias de las personas de más edad.

Los trabajadores de más edad reciben menos formación que los demás. El porcentaje de participación de los trabajadores en educación y formación desciende con la edad, y esta tendencia comienza en una fase temprana de la vida activa. Por tanto, para aumentar el nivel de formación que reciben los trabajadores de más edad es esencial invertir esta tendencia decreciente desde una fase temprana del ciclo vital: sólo el aprendizaje permanente puede garantizar que los trabajadores de más edad del futuro dispondrán de las aptitudes necesarias para adaptarse a los cambios del mercado de trabajo. El siguiente gráfico muestra que el porcentaje de trabajadores que participa en la educación y formación en cada grupo de edad sólo creció moderadamente en los últimos años, y que la evolución es demasiado lenta.

\section{Políticas del mercado de trabajo eficaces}

Para que los trabajadores puedan permanecer más tiempo en activo, es esencial prever los cambios y gestionar con éxito la reestructuración económica. En particular, los trabajadores de más edad tienen un riesgo mayor de abandonar el mercado de trabajo en caso de reestructuración o reducción de las empresas. Es preciso reforzar las políticas activas del mercado de trabajo a fin de prevenir el desempleo y ayudar a los trabajadores de más edad que pierdan su trabajo a permanecer en el mercado y encontrar otro empleo. A este respecto, revisten especial importancia los enfoques personalizados para atender las necesidades individuales, por 
ejemplo, a través de servicios de orientación, formación específica y sistemas de recolocación. Además, debe evitarse usar los regímenes de prestaciones por desempleo como vía alternativa a la jubilación anticipada para los trabajadores de más edad. Es preciso luchar contra esta sustitución entre regímenes de prestaciones mediante una activación de los regímenes, apoyando la contratación de trabajadores de más edad desempleados al mismo tiempo que se mantienen los derechos a las prestaciones por desempleo, y ofreciendo orientación que estimule la transición a otro empleo por cuenta ajena o por cuenta propia.

\section{Mejora de la calidad del empleo}

La higiene y seguridad en el trabajo, la organización del trabajo y la formación son dimensiones clave de la calidad del empleo. Como señala el informe Empleo en Europa 2002, la calidad del empleo es, en términos generales, crucial para volver a atraer hacia el mercado de trabajo a las personas de más edad y a aquellas que tienen responsabilidades asistenciales. Al mismo tiempo, las cifras de abandono del mercado de trabajo son hasta cuatro veces más importantes en el caso de los trabajadores de más edad que tienen empleos de baja calidad que para los que gozan de empleos de más calidad, aunque también representan más del doble de las de los jóvenes con empleos de baja calidad. En particular, unas condiciones contractuales flexibles deben ir acompañadas de formación continua u ofrecer oportunidades de ascenso para poder ayudar a que los trabajadores de más edad permanezcan en el mercado de trabajo o regresen a él.

\section{Referencias}

BAREA T. J.; GONZALEZ-PARAMO, B. Pensiones y prestaciones por desempleo. Bilbao: Fundación BBV, 1996. 
EUROSTAT. Disponible en: <http://epp.eurostat.ec.europa.eu/portal/page/portal/eurostat/home/>. Consultado el: 20 dic. 2009.

VOGLER-LUDWIG, K. Crisis managment for european labour markets. 2009. Disponible en: <http://www.mutual-learning-employment.net/labour marketresponses051909.html>. Consultado el: 20 dic. 2009.

Recibido: 01/02/2010

Recebido: 01/02/2010

Aprobado: 23/04/2010

Aprovado: 23/04/2010 